\title{
Understanding pH-Induced Softening of Feta Cheese During Storage at the Ultrastructural Level - A Structure-Function Case Study
}

\author{
Almut H. Vollmer ${ }^{1}$, Nabil N. Youssef ${ }^{2}$, James A. Powell ${ }^{3}$, Xiaojun Qi ${ }^{4}$ and Donald J. McMahon ${ }^{5}$ \\ 1., 2., 5. Department of Nutrition, Dietetics, and Food Sciences, Utah State University, Logan, USA \\ 3. Department of Mathematics and Statistics, Utah State University, Logan, USA \\ 5. Department of Computer Science, Utah State University, Logan, USA
}

Like biological tissue, the cheese we eat or see in the store is a dynamic system that is continuously changing structurally and functionally as a consequence of a complex interplay of biotic and abiotic factors. These changes start with the beginning of milk fermentation and continue through acid- or rennet-induced coagulation of the major milk proteins (caseins) to form a gel. Then, the minor milk proteins (whey) and water are expelled from the gel to form the curd. Depending on the cheese variety, the curd is handled in various ways. Finally, through the process of aging, or ripening, the cheese body develops its characteristic texture and flavor profile. In industry, these numerous macroscopic changes are closely monitored since a sudden or undesired change may cause economic loss.

We hypothesize that microscopic changes form the structural basis for the changes in physical attributes of cheese, such as cheese firmness. To understand the microscopic changes resulting from changes in the firmness of the cheese body we selected Feta cheese. Feta is a low-pH, rennet-set, brined cheese with a crumbly texture. This characteristic texture is reflected by an open microstructure in which the electron-dense casein matrix forms a loosely connected, sponge-like network trapping fat, starter bacteria, and water within (Figure 1a). It has been reported anecdotally that a change in $\mathrm{pH}$ at an early point during the cheese making process - before brining (submerging in an aqueous $\mathrm{NaCl}$ solution) induces softening of the cheese body during storage. The characteristically crumbly texture is consequently lost and the cheese becomes unmarketable. We therefore manufactured Feta cheese with a range of $\mathrm{pH}$ (A, control: $\mathrm{pH} 4.80 ; \mathrm{B}: 4.88 ; \mathrm{C}$ : 5.17) by experimentally modulating the overnight storage temperature and hence fermentation rate of the starter cultures in the cheese body before brining. Feta cheese was then ripened in $14 \%$ brine at $3^{\circ} \mathrm{C}$ for up to 4 months. For structural analysis, samples were taken at Days 1, 7, and 90, and processed for transmission electron microscopy (TEM). In addition, texture profile analysis (TPA) was performed on samples taken at Day 120 to quantitatively assess cheese body firmness. Firmness was measured by the maximum force to compress a 2 -cm cheese cube to $70 \%$ of its original height and the maximum force to shear a $2-\mathrm{cm}$ cheese cube at a speed of 30 $\mathrm{mm} / \mathrm{sec}$. Both measurements showed significant loss of firmness for higher-pH Feta cheese B and C. Concluding, data from TPA confirmed the effect of $\mathrm{pH}$ on aging cheese. Low-magnification TEM analysis indicated a progressive change in the organization of the protein matrix, particularly in the highest-pH cheese $\mathrm{C}$ (Figure $1 \mathrm{f}$ and e). At high magnification, fine proteinaceous material was first seen to dissociate from the periphery of the protein matrix (Figure 2; Day 1), then to aggregate within the liquid phase (Figure 2; Day 7), and finally to form a continuous, albeit less dense and inhomogeneous protein matrix surrounding globular fat inclusions (Figure 2; Day 90). High-magnification TEM also revealed a fundamental change in the protein matrix itself. With increasing $\mathrm{pH}$ and storage time, the protein matrix had lost the regular, finely granulated appearance and larger clusters had formed (Figure 1, inserts). To quantitate these changes, different image processing methods were used. Basic image analysis tools in FIJI (ImageJ) gave contradictory results. More advanced methods based on a customwritten script for cluster analysis in MatLab (MathWorks) also did not satisfactorily quantitate the 
differences which were so easily visually discernable in the casein matrix of the high-magnification micrographs. However, by applying a spatial autocorrelation function in MatLab, we were able to reliably quantitate the differences and corroborate our qualitative assessment.

By presenting a clear sequence of events, we argue that the above-described microstructural modifications of the casein network caused the softer texture of aged Feta cheese as a consequence of inadequate acidification before brining. We further demonstrated the power of image analysis but also the challenges of finding an appropriate metric for the quantification of differences in the morphology of the casein matrix. In summary, our study provides a compelling example of how structure and function are tied together in a complex food system and how TEM coupled with image analysis was able to precisely establish this relationship [1].

\section{References:}

[1] The authors acknowledge Leprino Foods Company for the support they have given to the Western Dairy Center at Utah State University to establish a Biostructure Center, and the Electron Microscopy Core Research Facility at the University of Utah for access to the transmission electron microscope.

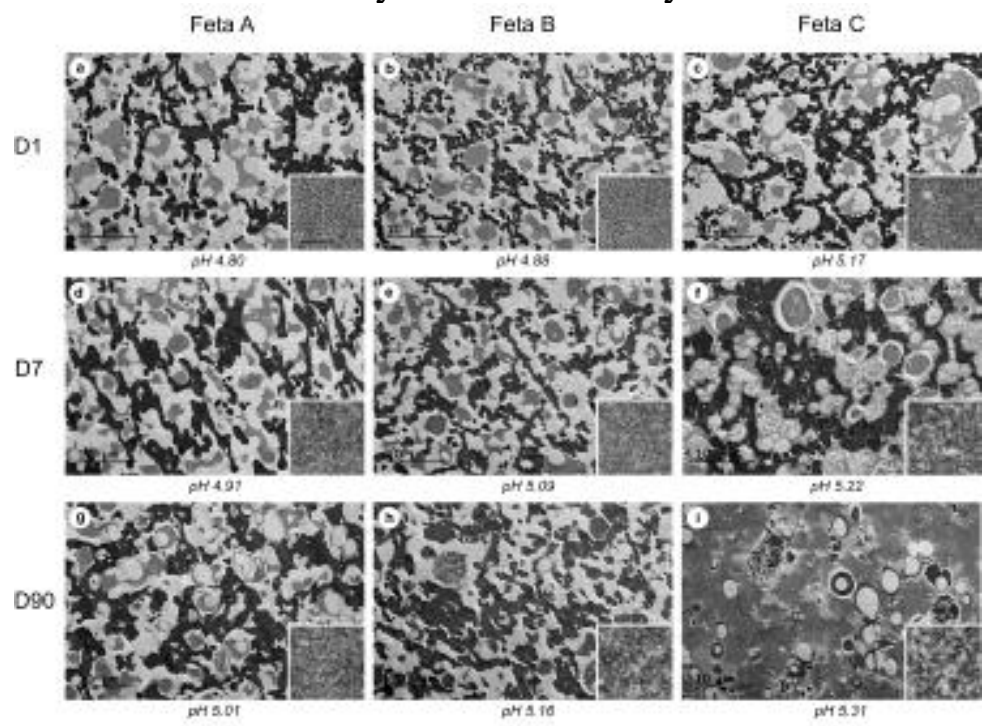

Figure 1. The microstructure of aging Feta cheese that differ in initial $\mathrm{pH}$ with A being the control at low magnification. Samples were taken at Day 1, 7, and 90 after brining. Scale bar $10 \mu \mathrm{m}$. Inserts show the protein matrix at high magnification. Scale bar $100 \mathrm{~nm}$.

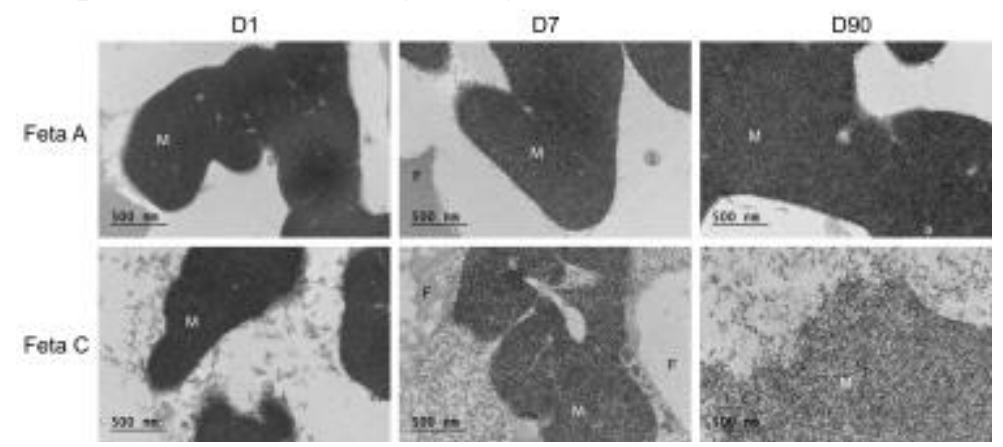

Figure 2: Progressive dissociation of the casein matrix (M) during storage in high-pH Feta cheese (C) compared to control (A). F, fat inclusions. Scale bar $500 \mathrm{~nm}$. 